\title{
Association Between Nurses' Years of Practice and Knowledge on Insulin Therapy at Edward Francis Small Teaching Hospital, the Gambia: A Cross-sectional Study
}

\author{
Tobiloba Oyejide Alex Omotosho ${ }^{1, ~}$, Haddy Tunkara-Bah ${ }^{1}$, Tomilayo Felicity Omotosho ${ }^{1}$, \\ Pateh Saho
}

${ }^{1}$ Department of Nursing and Reproductive Health, University of the Gambia, Banjul, The Gambia

${ }^{2}$ Department of Nursing, Edward Francis Small Teaching Hospital, Banjul, The Gambia

\section{Email address:}

ooalex@utg.edu.gm (T. O. A. Omotosho), htbah@utg.edu.gm (H. Tunkara-Bah),otfelicity@utg.edu.gm (T. F. Omotosho), pjksaho@yahoo.com (P. Saho)

${ }^{*}$ Corresponding author felex

\section{To cite this article:}

Tobiloba Oyejide Alex Omotosho, Haddy Tunkara-Bah, Tomilayo Felicity Omotosho, Pateh Saho. Association Between Nurses'Years of Practice and Knowledge on Insulin Therapy at Edward Francis Small Teaching Hospital, the Gambia: A Cross-sectional Study. International Journal of Diabetes and Endocrinology. Vol. 4, No. 2, 2019, pp. 49-56. doi: 10.11648/j.ijde.20190402.12

Received: April 20, 2019; Accepted: May 28, 2019; Published: June 11, 2019

\begin{abstract}
The purpose of this study was to measure the association between years of nursing practice and knowledge of insulin therapy among nurses in Edward Francis Small Teaching Hospital, The Gambia. A hospital-based descriptive crosssectional study was conducted among 127 randomly selected trained nurses. Data were collected using a self-administered questionnaire. Data were analysed using IBM SPSS version 20. A total of 127 nurses participated in this study but 118 completely filled and returned the questionnaires making a total response rate of $93 \%$. The mean years of experience of nurses was $3.86 \pm 4.051$ years with a minimum of 1 year $(20.3 \%)$ and maximum of 23 years $(0.8 \%)$. Most of the nurses were females $(\mathrm{n}=66,55.6 \%)$, had a diploma in nursing $(\mathrm{n}=75,63.6 \%)$ and working at the surgical department $(\mathrm{n}=47,39.8 \%)$ respectively. The majority $(\mathrm{n}=93,78.8 \%)$ of them had never attended an in-service training on management of diabetes. The majority of nurses rated their knowledge of diabetes as good $(n=72,61 \%)$. Despite this self-rating, $114(96.6 \%)$ of them would like to receive extra training on insulin therapy. Eighty-six percent $(n=102)$ of the nurses knew the normal range of fasting blood sugar level and $98.3 \%$ said that blood sugar level should be checked prior to administering insulin $(\mathrm{n}=116)$. However, most of them did not know that a blood sugar level of less than $70 \mathrm{mg} / \mathrm{dl}$ in a diabetic patient is classified as hypoglycaemia (n $=77$, $65.3 \%$ ). There were significant mean differences of the nurses' years of experience in relation to their willingness to attend an in-service training on DM management and knowledge of sign and symptoms of hypoglycaemia $(\mathrm{p}<0.05)$. The majority $(87.3 \%)$ and $(91.5 \%)$ of the nurses practiced injection site cleaning and priming of the insulin syringe respectively. However, $33.1 \%(n=39)$ of the nurses do not wash their hands and only $4.2 \%(n=5)$ of them reported checking for expiry date prior to giving insulin injection. In addition, $72.9 \%(\mathrm{n}=86)$ of the nurses reported administering insulin injection in the arm. The nurses with more than four years of practice were more knowledgeable on the requirements of effective insulin administration than those with two years or less practical experience. The findings of this study highlight the urgent need for an educational intervention on diabetes and insulin therapy for the nurses in EFSTH.
\end{abstract}

Keywords: Nurses, Knowledge, Insulin, Therapy, Diabetes, EFSTH, The Gambia

\section{Introduction}

Diabetes mellitus (DM) is a metabolic disorder of multiple etiologies characterized by chronic hyperglycemia with disturbances of carbohydrate, fat and protein metabolism, resulting from defects in insulin secretion, insulin action, or both [1]. DM has proved to be the most common global public chronic disease that is associated with serious long term effects and escalating healthcare costs [2]. Existing 
studies have revealed definite global increase in its incidence and prevalence [3-5]. The International Diabetes Federation [3] reported that the DM prevalence was 415 million people worldwide in 2015 and this number is going to increase to 642 million people by 2040 . This report continued to indicate that 15.5 million of the DM incidence was from the African Region, representing a regional prevalence of $3.3 \%$. The highest prevalence of diabetes in the Region is found in adults aged 55 to 64 [3-6]. The prevalence of diabetes is even higher in The Gambia estimated at 5.8\% [2].

The main purpose of DM treatment is to prevent the development of complications that worsen quality of life or even cause deaths. To achieve this, insulin therapy has been used to keep the blood sugar within normal range and prevent acute and chronic complications $[5,6]$. Insulin therapy is a lifesaving treatment for hyperglycaemic patients in the hospital setting. Insulin can be stored in a refrigerator for approximately 3 months or kept at room temperature (15$25^{\circ} \mathrm{C}$ ) for 28 days [6-8]. Health care professionals need to draw their attention to all areas relating to insulin treatment to achieve optimal glucose control in the blood $[9,10]$. Nurses are often the first point of contact for people seeking information on diabetes care [10] and therefore have a major role in administration of insulin as well as the responsibility of identifying the suspicious signs of hyperglycemia and hypoglycemia. Inadequate knowledge of nurses on insulin treatment can cause errors and several adverse outcomes. Errors related to insulin treatment and application includes problems with insulin administration timing, type as well as the route of administration or injection technique $[11,12]$. It is therefore expected that nurses should have information about how insulin works, when and why insulin is needed, how dosing regimens and how changes are made [13]. Therefore, the purpose of this study was to determine the level of knowledge of insulin therapy among nurses in Edward Francis Small Teaching Hospital, The Gambia. Search of the literature did not reveal any similar study conducted in The Gambia. Hence, this study is highly needed to fill this knowledge gap.

\section{Materials and Methods}

\subsection{Study Design}

A hospital-based descriptive cross-sectional study design was used.

\subsection{Study Setting}

The study was conducted at Edward Francis Small Teaching Hospital (EFSTH), Banjul, The Gambia. At EFSTH, there are two distinct categories of nurses, i.e., trained (Registered nurses) and untrained nurses (nurse assistants/attendants). Majority of the trained nurses are not specialized in any particular nursing specialization area and so are subject to rotation to various departments within the hospital according to hospital policy. For example a nurse working in a paediatric ward can be moved to an adult medical ward depending on demands. Nurse assistants have not undergone nurse training in a training institution but are trained on the job and serve assistants to the trained nurses.

\subsection{Target Population}

According to the staff records at EFSTH [14], 400 nurses were working in the hospital during the study period. The eligibility criteria for this study included all trained nurses working at EFSTH during the period of the data collection and consented to participate in the study. Nurses working in the wards of the various departments, for example the Surgical, Obstetric and Gynae, Medical and Accident and Emergency Departments were included because they do care for patients with diabetes and its complications such as management of diabetic foot ulcer and amputation, diabetes in pregnancy and diabetic coma. However, among the trained nurses working in Paediatric Department, only nurses working at the Paediatric Surgical Unit were included in the study since they do care for children with diabetes and within the age group of 7 to 14 years. Nurses working outside the ward setting or on leave were excluded from the study.

\subsection{Sampling Size and Sampling Technique}

The sample size was calculated using the Research Advisor Sampling Template, using a population size of 400 nurses in EFSTH, set at a confidence interval of $95 \%$ and a marginal error (degree of accuracy) of $5 \%$ to give a required sample size of 115 nurses. To make up for possible nonresponse and incompletely filled questionnaires, $10 \%$ of the calculated sample size was added to increase it to 127 respondents. These respondents were selected using simple random sampling. The EFSTH staff list was used as the sampling frame.

\subsection{Data Collection Tool}

The instrument used in this study was adapted from another study [1] with few alterations made to best suit The Gambian context but not to the detriment of the retention of important information the survey intends to provide to and from study participants. This structured questionnaire consists three parts, of which one part was dealing with the nurses' socio-demographic characteristics, while the other two parts were measuring the nurses' knowledge of DM and administration of insulin therapy such as insulin effects, complications of insulin treatment, insulin administration routes, insulin preservation, and hypoglycaemia indications and treatment.

\subsection{Scoring of the Questionnaire}

Part 2 which measured knowledge on diabetes had 5 questions and $20 \%$ was assigned to correct answer for each question. The total possible score was $100 \%$ and a score of 0 - 59 was graded as low knowledge and $60-100 \%$ as high knowledge.

Part 3 measuring knowledge on insulin administration had 13 questions. Each correct answer was scored 1 and incorrect 
answer scored 0 . The total percentage score on each item by individual respondent was calculated and $0-59 \%$ was categorized as low knowledge while $60 \%-100 \%$ was high knowledge.

\subsection{Validity and Reliability of Study Questionnaire}

The study tool was sent to three endocrinology and research experts to critique for comprehensiveness, representativeness and clarity so as to ensure it measures what it was purported to measure in the Gambian context. The questionnaire was pilot tested among ten nurses from Serekunda Hospital and the total reliability coefficient of the questionnaire was 0.81 .

\subsection{Data Collection}

Data was collected using self-administered method which lasted for one month (August, 2018). The researchers went from ward to ward distributing the questionnaires to the nurses. The researchers were available to respond to doubts or questions from the respondents and to ensure that they answered the questions individually.

\subsection{Data Analysis}

Data was analysed using IBM SPSS version 20. Categorical variables were presented as frequencies and percentages. Independent sample t-test was used to compare means between two groups. One way Analysis of variance (ANOVA) was used to compare means between more than two groups. The level of statistical significance was set at $\mathrm{p}<0.05$.

\subsection{Ethical Approval}

Approval to conduct the research was obtained from the Hospital's Research Review Board. The study respondents signed informed consent forms.

\section{Results}

\subsection{Demographic Characteristics of Study Respondents}

A total of 127 nurses participated in this study but 118 completely filled and returned the questionnaires making a total response rate of $93 \%$. The mean years of experience for nurses was $3.86 \pm 4.051$ years with a minimum of 1 year $(20.3 \%)$ and maximum of 23 years $(0.8 \%)$. As shown in Table 1 , most of the nurses were females $(n=66,55.6 \%)$, had a diploma in nursing $(\mathrm{n}=75,63.6 \%)$ and working at the surgical department $(n=47,39.8 \%)$ respectively. Majority $(n=93$, $78.8 \%$ ) of them had never attended an in-service training on management of diabetes. Table 1 also shows that the mean differences of nurses' years of experience by sociodemographic characteristics. The male nurses (mean = $4.21 \pm 4.620$ ) had longer years of work experience than their female counterparts. There was also a significant difference in their years of nursing experience between their highest level of education and those who said they had attended an in-service training on management of diabetes $(p<0.05)$. However, there was no statistically significant differences between their years of experiences and the different departments nurses worked in.

Table 1. Mean differences of nurses'years of experience by socio-demographic characteristics.

\begin{tabular}{llll}
\hline Socio-demographic characteristics & $\mathbf{N}=\mathbf{1 1 8}$ & $\mathbf{\%}$ & Years of experience Mean \pm SD \\
\hline Sex & & & P value \\
Male & 52 & 44.1 & $4.21 \pm 4.620$ \\
Female & 66 & 55.9 & $3.59 \pm 3.552$ \\
Educational Level & & & \\
Diploma & 75 & 63.6 & $3.77 \pm 3.941$ \\
University Degree & 42 & 35.6 & $3.57 \pm 3.125$ \\
Master degree & 1 & 0.8 & $23.00 \pm 0.0$ \\
Department & & & $3.45 \pm 3.680$ \\
Medicine & 29 & 24.6 & $3.85 \pm 4.102$ \\
Surgery & 47 & 39.8 & $2.40 \pm 1.075$ \\
Paediatrics & 10 & 8.5 & $4.60 \pm 5.066$ \\
Accident \&Emergency/ICU & 25 & 21.2 & $5.14 \pm 3.848$ \\
Obs\&Gyne & 7 & 5.9 & $0.001 *$ \\
Attended In-service DM training courses & & & $4.84 \pm 6.019$ \\
Yes & 25 & 21.2 & $2.60 \pm 2.330$ \\
No & 93 & 78.8 & \\
\hline
\end{tabular}

$* \mathrm{p}$ value $<0.05$ is significant

\subsection{Sources of Information on Diabetes and Insulin Therapy}

Sixty-two $(52.5 \%)$ of the nurses revealed that their most important source of information on DM and insulin treatment was the nursing training institutions while 9 (7.6\%) said seminars/workshops (Table 2).
Table 2. Source of information on DM and insulin treatment $(n=118)$.

\begin{tabular}{lll}
\hline Items & Frequency & Percentage (\%) \\
\hline None & 2 & 1.7 \\
Doctors & 9 & 7.6 \\
Nurses & 13 & 11.0 \\
Self & 15 & 12.7 \\
Nursing training institution & 62 & 52.5 \\
Seminar/workshop & 9 & 7.6 \\
Media & 8 & 6.8 \\
\hline
\end{tabular}




\subsection{Knowledge of Diabetes Among Nurses}

The majority of nurses rated their knowledge of diabetes as good $(\mathrm{n}=72,61 \%)$. Despite this self-rating, $114(96.6 \%)$ of them would like to receive extra training. The nurses with 1 year nursing experience $($ mean $=1.36 \pm 0.867)$ reported more that they would like to receive extra in-service training on diabetes management $(\mathrm{n}=114,96.6 \%)$ than those with 5 or more years of experience. Thus, there was a significant mean difference of the nurses' years of experience in relation to their willingness to attend an in-service training on DM management $(\mathrm{p}=0.026)$.

Eighty-six percent $(\mathrm{n}=102)$ of the nurses knew the normal range of fasting blood sugar level and $98.3 \%$ said that blood sugar level should be checked prior to administering insulin $(\mathrm{n}=116)$. However, most of them did not know that a blood sugar level of less than $70 \mathrm{mg} / \mathrm{dl}$ in a diabetic patient is classified as hypoglycaemia $(\mathrm{n}=77,65.3 \%)$. The most known signs of hypoglycaemia among the nurses were a feeling of a dry mouth $(n=37)$ and blackout $(n=36)$ whilst that of hyperglycaemia were dry mouth $(n=39)$ and frequent urination $(\mathrm{n}=30)$. Most of the nurses' general level of knowledge on diabetes was low $(\mathrm{n}=71,60.2 \%)$. However, the nurses with 4 more years of nursing experience were more likely to have high level of knowledge on diabetes than those with 2 or lesser years of experience $(\mathrm{p}=0.037)$.

Table 3. Mean differences of nurses' years of experience by knowledge of DM.

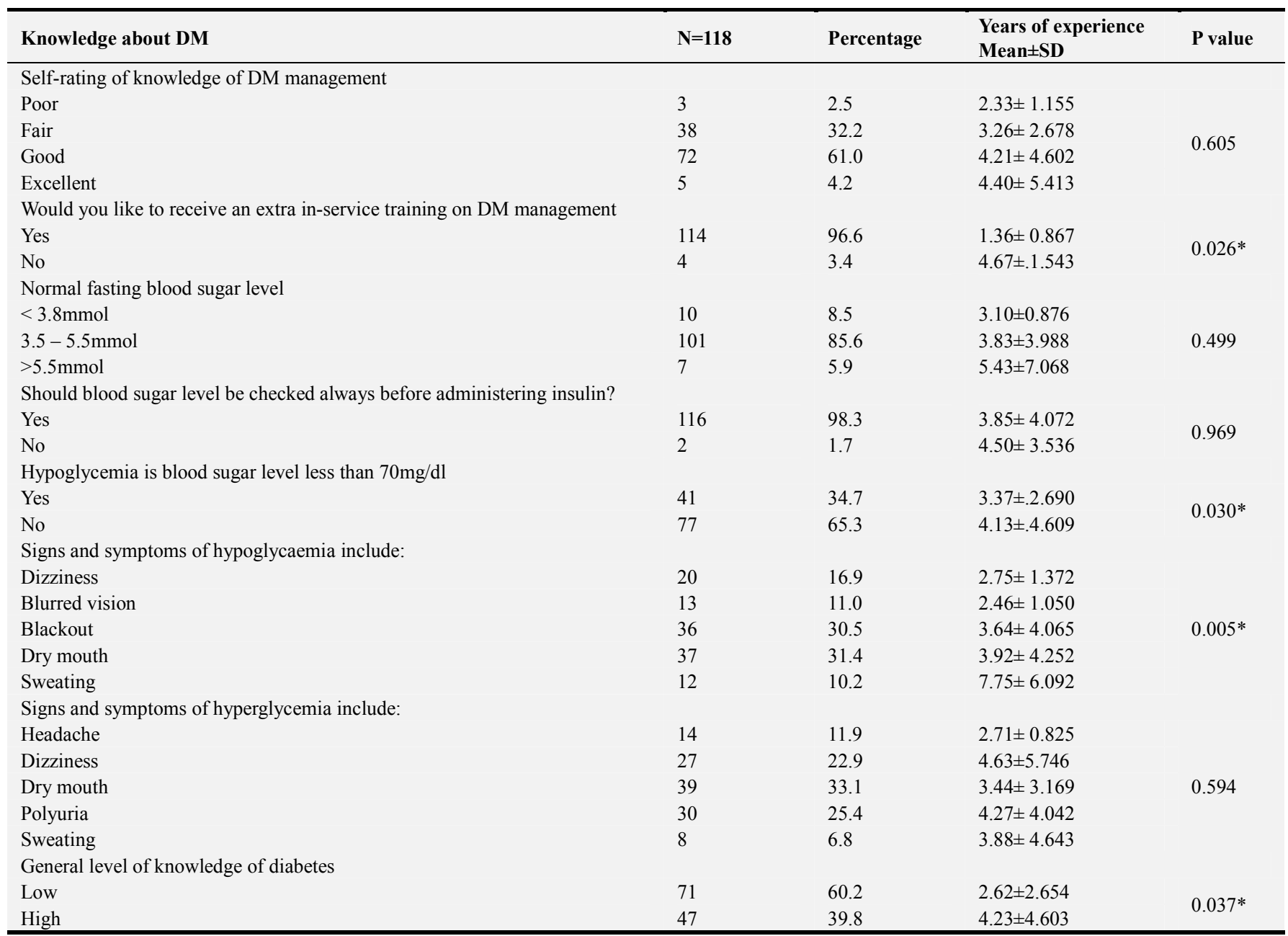

$* \mathrm{p}$ value $<0.05$ is significant

\subsection{Nurses' Knowledge of Insulin Therapy}

The results presented in Table 4 show that the majority $(87.3 \%)$ and $(91.5 \%)$ of the nurses practiced injection site cleaning and priming of the insulin syringe respectively. However, $33.1 \%(\mathrm{n}=39)$ of the nurses do not wash their hands and only $4.2 \%(\mathrm{n}=5)$ of them reported checking for expiry date prior to giving insulin injection. In addition,
$72.9 \%(\mathrm{n}=86)$ of the nurses reported administering insulin inject in the arm. The total knowledge of the nurses on insulin therapy was low $(\mathrm{n}=84 ; 74.6 \%)$. There were significant mean differences of the nurses' years of experience in relation to priming of the insulin syringe, washing of their hands, time of hand wash while giving insulin and the frequency of insulin syringe use $(\mathrm{p}<0.05)$. 
Table 4. Mean differences of nurses'years of experience by knowledge on implementation of insulin therapy.

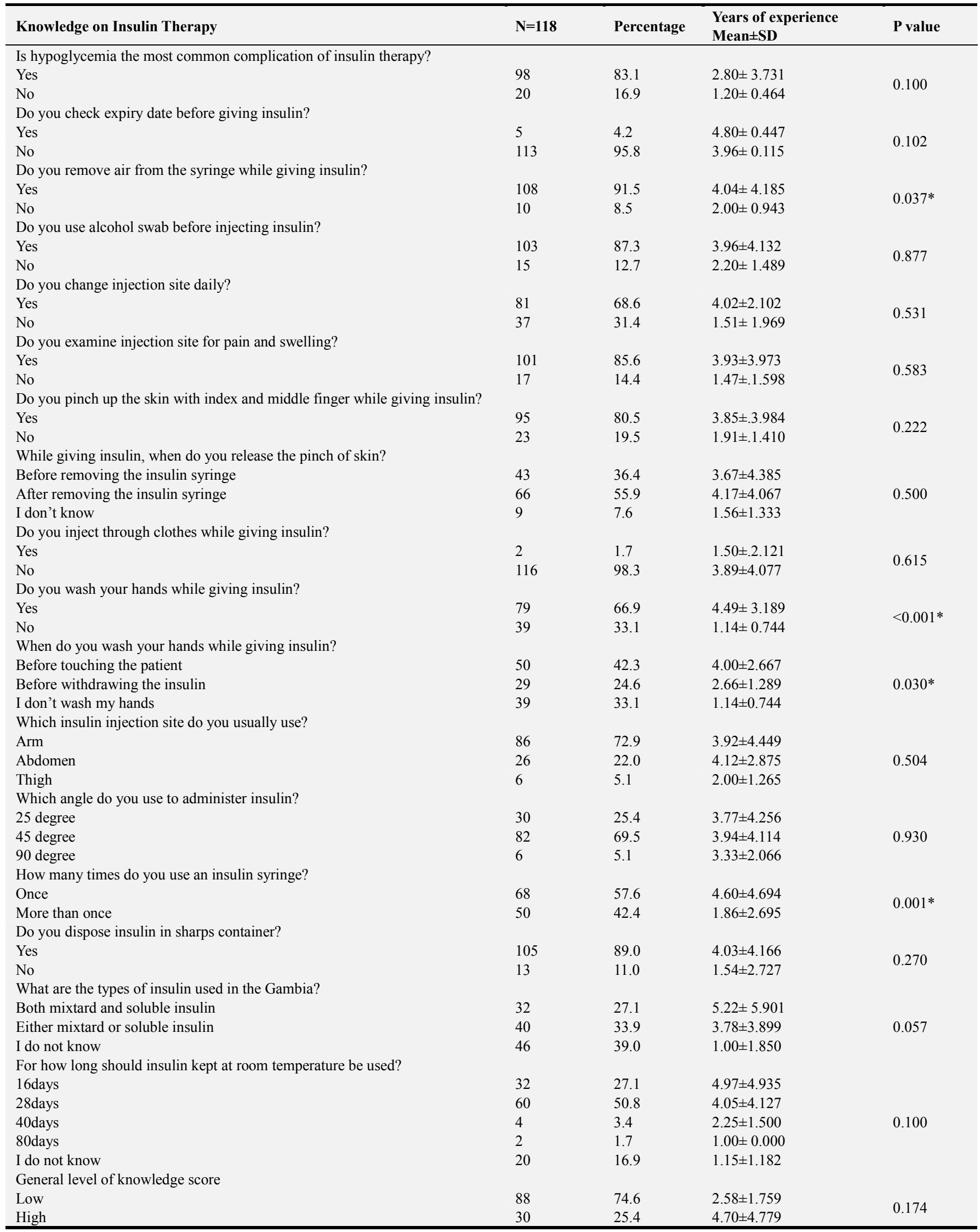

*p value $<0.05$ is significant 


\section{Discussion}

Most of the nurses had years of experience less than 5 . This could be attributed to the high turnover of nurses in The Gambia [16]. Similarly, Adhikari, et al., and Mohammed [15, 17] reported that younger and less experienced nurses contributed to suboptimal patient care. Nurses are crucial frontline healthcare professionals and are responsible for proper insulin administration for diabetics admitted in the hospital. Therefore, their knowledge about insulin treatment can help them to properly serve their main role as guardians of patient's safety. The success of diabetic mellitus (DM) treatment with insulin does not only depend on the type and dose of insulin administration but also in the technique of administration [1].

The finding that most of the nurses would like to have extra training on DM management is similar to the findings from the studies conducted by Chrysoula and Findlow $[9,18]$ that $84.1 \%$ and $94.8 \%$ of their respective respondents would like to be trained in the organization and application of insulin treatment. Furthermore, the nurses in this study reported that nursing training institutions were their most important source of information on insulin therapy. It could be inferred then that nurses with longer years or advanced training in any nursing institution were more likely to have better knowledge. Hence there was a significant differences between level knowledge of insulin therapy and years of experience.

In our study, most of the nurses could not ascertain what value a hypoglycaemic blood sugar should read in a patient and there was significant relationship between nurses' years of experience and sign and symptoms of hypoglycemia. Having adequate and comprehensive knowledge of diabetes and insulin therapy is important since nurses are responsible for giving health education on the use and complications of insulin to a patient upon their discharge from the hospital. According to the guidelines from the American Diabetes Association and International Foundation diabetes [19], specialized knowledge on insulin-dependent diabetes mellitus and skills are required by nurses.

It is important that healthcare practitioners check the manufacturer's recommendation for proper storage conditions [20] because storage of insulin at inappropriate temperature decreases the potency and pharmacological action of insulin [21-22]. Most of the nurses in this study were aware of the recommendations for insulin storage at room temperature. Contrasting findings were reported among nurses in Nepal [15] and Kenya [23].

There are three areas that generally could be used for injecting: thighs, abdomen and buttocks. Injecting into the arms is always avoided unless advised by the diabetes team because there is an increased risk of hypoglycaemia when injecting into the arms as these injections might become intra-muscular rather than subcutaneous [24]. However, majority of the nurses in this study reported using the arm as site of injecting insulin. Wrong route of administration was found to be a common error when administering insulin in a study conducted in Kenya [23]. Before injecting a patient with insulin, it is recommended that nurses follow the principles of anti-septic and non-touch technique [23]. Even though majority of the nurses claimed that they use alcohol swab to clean the injection site before injecting, there were significant statistical difference in years of experience with performing hand hygiene. Such practice could lead to unpredictable clinical outcomes [18-20] and is against the policy for safe administration of insulin [21-23]. There was significant relationship between the nurses' years of experience and frequency of insulin syringe re-use. Needle re-use is usually associated with contamination, infection and increased risk of lipo-hypertrophy. The risk of lipohypertrophy significantly increases with the use of needles for more than five times [25].

\section{Strengths and Limitation of the Study}

Knowledge of proper use of insulin is indispensable and essential to patient safety. To our knowledge, this is the first study in The Gambia to report the knowledge of nurses on insulin therapy. However, this study was limited to a single centre which is the only teaching hospital in the country as at the time of the study and thus the findings might not be generalized. This study used self-administered questionnaire. In the knowledge section, most of the responses had 'yes or no' option which could cause a high risk for guessing.

\section{Conclusions}

This study investigated the association between years of nursing practice and knowledge of insulin therapy among nurses in Edward Francis Small Teaching Hospital. The general knowledge levels of the nurses on DM and insulin therapy were low. The findings identified the need for improvement in the knowledge and practice of care of diabetic patients among the nurses with less practical experience. Inadequate knowledge on diabetes management among nurses can lead to reduced knowledge of the patients as a result of inadequate health education and invariable poor health outcome. This can cause undermined confidence of patients on nurses and thus reduce the confidence of patients in the health system.

\section{Recommendations}

The results of this study highlight the need for urgent educational intervention for the nurses with less than 2 years practical experience on the application of insulin therapy. Thus, it is important to develop a plan for continuing education and organize training programs to improve the knowledge and skills of nurses on the care of people with diabetes in EFSTH. From the participants response to hand washing practice, it is imperative that studies be conducted on infection control and hand hygiene practice of the nurses in this hospital. 


\section{List of Abbreviations}

DM - Diabetes Mellitus

EFSTH - Edward Francis Small Teaching Hospital

\section{Consent for Publication}

All the authors consented for the publication of this article.

\section{Availability of Data and Material}

The data that supports the findings of the current study are available from the corresponding author upon reasonable request.

\section{Competing Interests}

The authors declare that they have no competing interests.

\section{Funding}

This research did not receive funding from any source.

\section{Authors' Contributions}

Tobiloba Oyejide Alex Omotosho, Tomilayo F. Omotosho, and Pateh Saho took part in conceptualization. Tobiloba Oyejide Alex Omotosho and Tomilayo F. Omotosho took part in recruitment of participants, data collection, compilation and entry. Tobiloba Oyejide Alex Omotosho wrote the manuscript. Tobiloba Oyejide Alex Omotosho and Haddy Tunkara-Bah analyzed and interpreted data. Haddy TunkaraBah reviewed it critically for important intellectual content and gave approval for publication. All the authors read and approved the final manuscript.

\section{Acknowledgements}

Appreciation goes to Dr. Musa Touray, senior lecturer, School of Medicine and Allied Health Science for his assistance with the data analysis.

\section{References}

[1] Alwan AH, Alhusuny A. Knowledge and practice about diabetes mellitus and insulin injection technique among nurses in general hospitals, Al-Hillah City. IOSR J Dental and Med Sci. 2014; 13 (9): 28-32.

[2] World Health Organization (WHO): World Health Day Beat diabetes. http://www.who.int/diabetes/en/(2016). Accessed 20 October 2018.

[3] International Diabetes Federation (IDF): http://www.diabetesatlas.org/ (2016). Accessed 22 October 2018.

[4] Kirtland KA, Li YF, Geiss LS, Thompson TJ. State-specific incidence of diabetes among adults-participating states, 19951997 and 2005-2007. MMWR Morb Mortal Wkly Rep. 2008; 57 (43): 1169-1173.
[5] Saygılı U, K1lıç SP. Determination of knowledge levels of nurses on insulin applications. International Journal of Diabetes and Endocrinology. 2017; 2 (4): 50-54. doi: 10.11648/j.ijde.20170204.11.

[6] International Diabetes Federation (IDF): Regional prevalence of diabetes. https://www.idf.org/our-network/regionsmembers/africa/welcome.html/ (2017). Accessed 22 October 2018.

[7] Avdal E, Kızılc1 S. Concept analysis of diabetes and lack of self-efficacy nursing theory. Dokuz Eylül University School of Nursing Electronic Journal. 2010; 3 (3): 164-168.

[8] Shu AD, Myers MG, Shoelson SE. Pharmacology of endocrine pancreas. In: Golan DE, Tashjian Jr AH, Armstrong EJ, Armstrong AW. Editors. Principles of pharmacology the pathophysiologic basis of drug therapy. 2nd ed. New Delhi: Wolters Kluwer (India); 2008.

[9] Chrysoula D, Victoria A, Anna P. Assessment of insulin treatment-related knowledge among nurses in pediatric hospitals. IOSR Journal of Pharmacy. 2016; 6 (4): 20-25.

[10] Derr RL, Sivanandy MS, Bronich-Hall L, Rodriguez A. Insulin-related knowledge among health care professionals in internal medicine. Diabetes spectrum, Baltimore, United States of America. 2007; 20 (3): 177-185.

[11] Down S, Kirkland F. Injection technique in insulin therapy. Nursing Times. 2012; 108 (10): 20-21.

[12] Wakefield PL, Wilson MA. Enhancing nurses' knowledge regarding the complex care of hospitalized patients on insulin. Journal for Nurses in Professional Development. 2014; 30 (4): 174-180.

[13] Özcan Ş. Current practices in insulin therapy: recommendations for reducing insulin delivery failures. Journal of Education and Research in Nursing. 2007; 4 (2): 22-28.

[14] Edward Francis Small Teaching Hospital (EFSTH): Staff Record (Unpublished data). Banjul, The Gambia. 2018.

[15] Adhikari S, Poudel RS, Rajbanshi L, Shrestha S. Assessment of insulin injection practice of nurses working in a tertiary healthcare center of Nepal". Hindawi: Nursing Research and Practice. 2018; Article ID 9375067.

[16] Saho P. Factors Influencing the High Attrition of Nurses at Edward Francis Small Teaching Hospital" (Unpublished Masters Thesis). Presented in Partial Fulfilment of the Requirements for the Award of the Master of Science Degree in Nursing. University of the Gambia, Banjul, The Gambia. 2011.

[17] Mohammed MA, Hafez MZ. Nurses' knowledge, skill and attitudes toward insulin errors for diabetic critically ill patients. AAMJ. 2013; 11: 4.

[18] Findlow ML, McDowell RS. Determining registered nurses knowledge of diabetes mellitus- nurses knowledge. Journal of Diabetes Nursing. 2002; 6 (6): 170-175.

[19] American Diabetes Association (ADA): National standards for diabetes self-management education. Diabetes Care. 2009; 32: 13-61.

[20] Frid AH, Kreugel G, Grassi G. New Insulin Delivery Recommendations. Mayo Clinic Proceedings. 2016; 91 (9): $1231-1255$. 
[21] InDependent Diabetes Trust (IDDT): Storing insulin: looking after your insulin and injecting devices. Northampton, NN1 4XS, United Kingdom. 2018.

[22] Vimalavathini R, Gitanjali B. Effect of temperature on the potency \& pharmacological action of insulin. Indian Journal of Medical Research. 2009; 130 (2): 166-169.

[23] Kedogo EK. Impact of insulin storage and administration technique on glycated heamoglobin among adult diabetic patients in kenyatta national hospital. A dissertation submitted in partial fulfillment for the requirements of the degree of master of pharmacy in clinical pharmacy of University of
Nairobi. November 2015.

[24] Razzaq-Sheikh J. Policy for the safe administration of insulin. East Chesire NHS Trust. 2016. http://www.eastcheshire.nhs.uk/About-TheTrust/policies/I/Insulin\%20\%20Safe $\% 20$ administration $\% 20$ of \%20-\%20ECT2544.pdf. Accessed on December 31, 2018.

[25] Mushtaq MA. Study of insulin injection technique amongst the nursing staff'. Pak J Med Sci. 2006; 22 (3): 310. 